\title{
INTEGRAL SPRINGER THEOREM FOR QUATERNIONIC FORMS
}

\author{
LUIS ARENAS-CARMONA
}

\begin{abstract}
J. S. Hsia has conjectured an arithmetical version of Springer Theorem, which states that no two spinor genera in the same genus of integral quadratic forms become identified over an odd degree extension. In this paper we prove by examples that the corresponding result for quaternionic skewhermitian forms does not hold in full generality. We prove that it does hold for unimodular skew-hermitian lattices under all extensions and for lattices whose discriminant is relatively prime to 2 under Galois extensions.
\end{abstract}

\section{$\S 1$. Introduction}

If $D$ is a ring with involution $x \mapsto \bar{x}$, an $n$-ary skew-hermitian form over $D$ is a map $h: D^{n} \times D^{n} \rightarrow D$ that is $D$-linear in the first variable and satisfies the identity $h(x, y)=-\overline{h(y, x)}$. Equivalently

$$
h\left(x_{1}, \ldots, x_{n} ; y_{1}, \ldots, y_{n}\right)=\sum_{i, j=1}^{n} x_{i} a_{i, j} \overline{y_{j}}, \quad a_{i, j}=-\overline{a_{j, i}} \in D .
$$

Using (1) it is possible to extend a skew-hermitian form to any ring with involution containing $D$. If $k$ is a number field and $D$ is a quaternion algebra over $k$, these forms share many algebraic and arithmetical properties of symmetric bilinear forms over $k$. If $D$ is not a division algebra there is a natural correspondence between skew-hermitian forms and symmetric bilinear forms over $k$ as we describe in Section 3. Certainly, this applies when the form is extended to $D_{K}=D \otimes_{k} K$ for some well chosen quadratic extension $K / k$. It is natural, therefore, to expect that many concepts in the theory of symmetric bilinear forms [14] have their analogs in the theory of skew-hermitian forms. In this paper we study the existence of an analog of the Arithmetical Springer Theorem proved by J. S. Hsia [9], [8], [11].

Received January 5, 2006.

2000 Mathematics Subject Classification: 11E41, 11E08, 11E12.

Supported by Fondecyt, Grant No. 1040227. 
Let $\mathcal{O}$ be the ring of integers of the number field $k$. Two symmetric bilinear forms $q, r: \mathcal{O}^{n} \times \mathcal{O}^{n} \rightarrow \mathcal{O}$ are in the same class if there is an invertible linear substitution $l$ with coefficients in $\mathcal{O}$, i.e., a linear map $l$ : $\mathcal{O}^{n} \rightarrow \mathcal{O}^{n}$, satisfying

$$
q(x, y)=r[l(x), l(y)]
$$

Two forms are said to be in the same genus if (2) is satisfied for an invertible linear substitution $l=l_{v}$ with coefficients in the completion $\mathcal{O}_{v}$ for every place $v$ of $k$, including the archimedean places if we define $\mathcal{O}_{v}=k_{v}$ when $v$ is archimedean. The problem of determining the number of classes in a genus is hard, but when the form is indefinite at some archimedean place it can be reduced to the much simpler problem of counting spinor genera in a genus [14]. The theory of spinor genera is the abelian part of the theory and depends on the image of the spinor norm. It has been connected to the machinery of class field theory through the spinor class field, a multiple quadratic extension that encodes information about the form [9], [11]. Extensive computations of spinor norm images for integral symmetric bilinear forms exist in the current literature [12], [7]. These computations have been used to study the following question [11]: Can two different spinor genera in the same genus become equal under an odd degree algebraic extension? In the indefinite case, this is another way of asking whether (2) can hold for a linear substitution $l$ with coefficients in $\mathcal{O}_{K}$, the ring of integers of a field $K$, where $K / k$ is an extension of odd degree. A negative answer to this question can be considered an arithmetical version of Springer Theorem. So far, this is known to be the case whenever 2 is unramified in the base field $k$ [11]. The following partial generalization of this result is also known [11]: The number of spinor genera in a genus is nondecreasing under an extension that is linearly disjoint to the spinor class field.

In this paper, we study the corresponding question when Integral symmetric bilinear forms are replaced by skew-hermitian forms over a maximal order $\mathfrak{D}$ of a quaternion division algebra $D$ over $k$ [5]. Two skew-hermitian forms $q$ and $r$ are in the same class if (2) is satisfied for some left $\mathfrak{D}$-linear invertible substitution $l$ with coefficients in $\mathfrak{D}$. We say that two skewhermitian forms are in the same genus if:

- (2) is satisfied for an invertible linear substitution $l=l_{v}$ with coefficients in the completion $\mathfrak{D}_{v}$ for every finite place $v$ of $k$. 
- (2) is satisfied for an invertible linear transformation $l$ with coefficients in the $k$-algebra $D$.

The last condition is trivial for symmetric bilinear forms because of the Hasse principle. This is not the case for skew-hermitian forms ([13], p. 138). The concept of spinor genus [5] and spinor class field [1] also extend to this setting. Some computations of the image of the spinor norm for this case are already available [5], [2]. If (2) is satisfied for some linear substitution $l$ with coefficients in a maximal order $\mathfrak{D}^{\prime}$ of $D_{K}$ containing $\mathfrak{D}$ we say that $q$ and $r$ are identified in $\mathfrak{D}^{\prime}$. In this paper we exhibit counterexamples to a quaternionic skew-hermitian analog to the Arithmetical Springer Theorem mentioned before, namely:

THEOREM 1. There exists a quaternion $\mathbb{Q}$-algebra D, a maximal order $\mathfrak{D}$ of $D$, a cubic extension $K$ of $\mathbb{Q}$, and binary skew-hermitian forms $h$, $h_{1}$ over $\mathfrak{D}$ that are in the same genus but different spinor genus, and are identified in $\mathfrak{D}^{\prime}$ for any maximal order $\mathfrak{D}^{\prime}$ of $D_{K}$ containing $\mathfrak{D}$.

THEOREM 2. There exists a quaternion $\mathbb{Q}$-algebra $D$, a maximal order $\mathfrak{D}$ of $D$, a binary skew-hermitian form $h$ on $D$, and a quadratic extension $K$ of $\mathbb{Q}$ that is linearly disjoint to the spinor class field of $h$, that satisfy the following:

- The genus of h has two spinor genera.

- The genus of the extension of $h$ to $\mathfrak{D}^{\prime}$ has only one spinor genus for any maximal order $\mathfrak{D}^{\prime}$ of $D_{K}$ containing $\mathfrak{D}$.

Theorems 1 and 2 are proved by explicit constructions. On the other hand, the analog of the arithmetical springer Theorem still holds under some restrictions. In Theorems $3-5 K / k$ is a finite extension of number fields, $D$ is a quaternion algebra over $k, \mathfrak{D}$ is a maximal order of $D$ and $\mathfrak{D}^{\prime}$ is a maximal order of $D_{K}$ containing $\mathfrak{D}$. Let $h$ denote an $n$-ary skewhermitian form over $\mathfrak{D}$, where $n \geq 2$, and let $h^{\prime}$ be its extension to $\mathfrak{D}^{\prime}$. The discriminant of $h$ is the reduced norm of the matrix $\left(a_{i, j}\right)_{i, j}$ in (1). A form $h$ over the ring $\mathfrak{D}\left(\operatorname{resp} . \mathfrak{D}_{v}\right)$ is unimodular if its discriminant is a unit of $\mathcal{O}$ $\left(\operatorname{resp} . \mathcal{O}_{v}\right)$.

TheOREM 3. Assume $h$ is unimodular. Two lattices in different spinor genera in the genus of $h$ cannot be identified in $\mathfrak{D}^{\prime}$ if $[K: k]$ is odd. The 
number of spinor genera in the genus of $h$ is not bigger that the number of spinor genera in the genus of $h^{\prime}$ whenever $K$ is linearly disjoint from the spinor class field of $h$.

THEOREM 4. Assume that the discriminant of $h$ is relatively prime to 2. Then two lattices in different spinor genera in the genus of $h$ cannot be identified in $\mathfrak{D}^{\prime}$ if $K / k$ is a Galois extension of odd degree.

A skew-hermitian form is diagonalizable if it can be put in the form (1) where $a_{i, j}=0$ for $i \neq j$. In this case we write $a_{i}$ instead of $a_{i, i}$. For any non-archimedean place $v$, let $\Delta_{v}$ be a unit of maximal quadratic defect [14], and let $\nu$ be the normalized valuation in $k_{v}$.

THEOREM 5. Two lattices in different spinor genera in the genus of $h$ cannot be identified in $\mathfrak{D}^{\prime}$ if $K / k$ is an odd degree Galois extension, provided that for every dyadic local place $v$ of $k$ at least one of the following conditions holds:

1. The algebra $D_{v}$ is not a division algebra and either $k_{v} / \mathbb{Q}_{2}$ is unramified, or $h$ is a unimodular form.

2. $v$ splits completely in $K / k$.

3. The algebra $D_{v}$ is a division algebra and the extension $h_{v}$ of $h$ to $D_{v}$ is not in any of the three following exceptional cases:

- 2 ramifies in $k / \mathbb{Q}$ and $h_{v}$ is not diagonalizable.

- $h_{v}$ is diagonalizable. Also $a_{m}^{2}=\lambda_{m}^{2} \beta$, for $m=1, \ldots, n$, where $\beta$ is a unit satisfying $\beta \notin \Delta\left(k_{v}^{*}\right)^{2}$. Furthermore, $\nu\left(\lambda_{m}\right)<\nu\left(\lambda_{m+1}\right)$, but for some $m$ we have $\nu\left(16 \lambda_{m}\right) \geq \nu\left(\lambda_{m+1}\right)$.

- $h_{v}$ is diagonalizable. Also $a_{m}^{2}=\lambda_{m}^{2} \beta$, for $m=1, \ldots, n$, where $\beta$ is a prime element. Furthermore, $\nu\left(4 \lambda_{m}\right) \leq \nu\left(\lambda_{m+1}\right)$ for all $m$, but for some $m$ we have $\nu\left(16 \lambda_{m}\right) \geq \nu\left(\lambda_{m+1}\right)$.

The exceptional cases are those in which the image of the spinor norm is unknown. It is likely, as far as we know, that the above result holds with only the Galois condition. The Galois condition cannot be eliminated even in Theorem 4. This happens because the norm principle [8] fails to extend to our setting in the case of local extensions of even degree. In Section 5 we give a description of the local lattices and extensions that exhibit this phenomenon in the non-dyadic case. 


\section{§2. Lattice extensions and the norm principle}

In this section we exhibit the relation between the norm principle and extension properties of genera and spinor genera. We do not assume in this section that $G$ is a unitary group for the sake of generality, although this is the only case that is used in this work.

Let $V$ be a vector space over a number field $k$, and let $G \subseteq \mathrm{GL}_{k}(V)$ be a semisimple linear algebraic group [16]. For any field $E$ containing $k$, we denote by $G_{E}$ the set of $E$-points of $G$. Let $F$ be the fundamental group of $G$ and $\widetilde{G}$ the universal cover of $G$ ([16], p. 63). The spinor norm on $G_{E}$ is the cohomology map $\theta_{E}: G_{E} \rightarrow H^{1}(E, F)$, defined by the short exact sequence $F \hookrightarrow \widetilde{G} \rightarrow G([16]$, p. 72$)$. The kernel of the spinor norm is denoted $G_{E}^{\prime}$.

Let $\mathcal{O}$ be the ring of integers of $k$ and $\Lambda$ an $\mathcal{O}$-lattice in $V$. The class $\operatorname{cls}(\Lambda, G, k)$ is the $G$-orbit of $\Lambda$. A lattice $L$ is in the genus gen $(\Lambda, G, k)$ if its localization $L_{v}$ is in the same $G_{k_{v}}$-orbit as the localization $\Lambda_{v}$ for any finite place $v$. Equivalently, $L$ is in $\operatorname{gen}(\Lambda, G, k)$ if $L$ is in the $G_{\mathbb{A}^{-}}$ orbit of $\Lambda$, for the usual action of the group $G_{\mathbb{A}}$ of adelic points of $G$ on the set of lattices in $V$ [1], [14], [16]. A lattice $L$ is in the spinor genus $\operatorname{spn}(\Lambda, G, k)$ if there exists a lattice $M$ that is in the same $G$-orbit as $L$ and whose localizations $M_{v}$ is in the same $G_{k_{v}}^{\prime}$-orbit as $\Lambda_{v}$ for any finite place $v$. Equivalently, $L$ is in $\operatorname{spn}(\Lambda, G, k)$ if $L$ is in the $G_{k} G_{\mathbb{A}}^{\prime}$-orbit of $\Lambda$, where $G_{\mathbb{A}}^{\prime}$ is the subgroup of $G_{\mathbb{A}}$ of elements $g$ whose local coordinate $g_{v}$ has trivial spinor norm for all $v$. The set of classes in a genus is classified by the set of double cossets $G_{k} \backslash G_{\mathbb{A}} / G_{\mathbb{A}}^{\Lambda}$ [1], where $G_{\mathbb{A}}^{\Lambda}=\prod_{v} G_{k_{v}}^{\Lambda}$ is the stabilizer of $\Lambda$ in $G_{\mathbb{A}}$. If $G$ is non-compact at some infinite place the concepts of class and spinor genus coincide, but clasifying forms up to spinor genera is simpler, since the set of spinor genera in a genus are in one-to-one correspondence with the quotient group $\Theta\left(G_{\mathbb{A}}\right) / \Theta\left(G_{\AA}^{\Lambda} G_{k}\right)$, where $\Theta$ is the restriction to the adelic group $G_{\mathbb{A}}$ of the product function $\prod_{v} \theta_{k_{v}}: \prod_{v} G_{k_{v}} \rightarrow \prod_{v} H^{1}\left(k_{v}, F\right)$. The set $I_{v}(\Lambda, k)=\theta_{k_{v}}\left(G_{k_{v}}^{\Lambda}\right)$ is called the local image of the spinor norm. The set $I(\Lambda, k)=\prod_{v} I_{v}(\Lambda, k)=\Theta\left(G_{\AA}^{\Lambda}\right)$ is called the global image of the spinor norm. We use the convention that $\Lambda_{v}=V_{v}$ if $v$ is archimedean.

Let $K / k$ be a finite extension of number fields. Note that the adelic group $G_{\mathbb{A}}$ embeds in the adelic group $G_{\mathbb{A}_{K}}$ by embedding each $G_{k_{v}}$ diagonally in the product of the groups $G_{K_{w}}$ for all $w$ over $v$. In particular $G_{\mathbb{A}}$ acts naturally on the set of lattices in $V_{K}$. For any place $w$ of $K$ let $v$ denote the corresponding place of $k$ and let $N_{w}: H^{1}\left(K_{w}, F\right) \rightarrow H^{1}\left(k_{v}, F\right)$ be the transfer map [6]. Let $N: \prod_{w} H^{1}\left(K_{w}, F\right) \rightarrow \prod_{v} H^{1}\left(k_{v}, F\right)$ be the function defined locally by $N(a)_{v}=\prod_{w \mid v} N_{w}\left(a_{w}\right)$. Let $X$ be a set of lattices in $V$ 
that is closed under genus equivalence. A correspondence $\Lambda \mapsto \psi(\Lambda)$ that associates a lattice in $V_{K}$ to every lattice in $X$ and such that $\psi(\sigma \Lambda)=\sigma \psi(\Lambda)$ for every $\sigma \in G_{\mathbb{A}}$ is called an extension rule. If $N[I(\psi(\Lambda), K)] \subseteq I(\Lambda, k)$ we say that the extension rule $\psi$ satisfy the norm principle for $\Lambda$ under the extension $K / k$.

PROPOSITION 2.1. If the extension rule $\psi$ satisfy the norm principle for $\Lambda$ under $K / k$, and $[K: k]$ is relatively prime to $|F|$, then for any lattice $L$ in the same genus as $\Lambda$, the extensions $\psi(\Lambda)$ and $\psi(L)$ are spinor equivalent if and only if the original lattices $\Lambda$ and $L$ are spinor equivalent.

Proof. Let $\Psi_{k}=\Theta\left(G_{\mathbb{A}}\right)$, and let $\Omega_{k}$ be the image of $H^{1}(k, F)$ in $\prod_{v} H^{1}\left(k_{v}, F\right)$. Because of the Hasse principle for simply connected semisimple algebraic groups ([16], p. 286), if we regard $G_{k}$ as a subgroup of $G_{\mathbb{A}}$, then $\Theta\left(G_{k}\right)=\Psi_{k} \cap \Omega_{k}$, hence

$$
G_{\mathbb{A}} / G_{\mathbb{A}}^{\prime} G_{k} G_{\mathbb{A}}^{\Lambda} \cong \Psi_{k} /\left(\Psi_{k} \cap \Omega_{k}\right) I(\Lambda, k)
$$

The spinor genus of $L$ corresponds to the coset of $\sigma \in G_{\mathbb{A}}$ satisfying $\sigma \Lambda=L$. If we regard $\sigma$ as an element of the adelic group $G_{\mathbb{A}_{K}}$ over $K$, then the class of $\sigma$ over $K$ corresponds to the spinor genus of $\psi(L)$. Hence it suffices to prove that if $r: \prod_{v} H^{1}\left(k_{v}, F\right) \rightarrow \prod_{w} H^{1}\left(K_{w}, F\right)$ is the restriction map, then $r^{-1}\left[\Omega_{K} I(\Lambda, K)\right] \subseteq \Omega_{k} I(\Lambda, k)$. This follows since the composition $N \circ r$ is multiplication by $[L: K]([6]$, p. 82$)$, hence it is invertible in the cohomology groups of $F$, which have exponent $|F|$.

Assume from now on that $F$ is isomorphic as a $\mathrm{Gal}(\bar{k} / k)$-module to the group $\mu_{n}$ of $n$-th roots of unity. Then $\Omega_{k}=H^{1}(k, F)=k^{*} / k^{* n}$, and the quotient $J_{k} / J_{k}^{n}$ of the idele group $J_{k}$ is contained in $\prod_{v} H^{1}\left(k_{v}, F\right)=$ $\prod_{v} k_{v}^{*} / k_{v}^{* n}$. In this case it is known ([15], Lemma 13) that $\Psi_{k} \subseteq J_{k} / J_{k}^{n}$. Since the spinor norm is surjective over non-archimedean local fields ([16], p. 284), it follows by weak approximation that $\Psi_{k} \Omega_{k}=J_{k} / J_{k}^{n}$, whence

$$
\Psi_{k} /\left(\Psi_{k} \cap \Omega_{k}\right) I(\Lambda, k) \cong J_{k} / k^{*} H(\Lambda, k),
$$

where $H(\Lambda, k)$ is the preimage in $J_{k}$ of $I(\Lambda, k)$. The class field corresponding to $k^{*} H(\Lambda, k)$ is the spinor class field $\Sigma_{\Lambda}[1]$. The condition $N[H(\psi(\Lambda), K)] \subseteq$ $H(\Lambda, k)$ implies that $\Sigma_{\Lambda}$ is contained in $\Sigma_{\psi(\Lambda)}$. Next proposition follows: 
Proposition 2.2. If $K \cap \Sigma_{\Lambda}=k$ and the extension rule $\psi$ satisfies the norm principle for $\Lambda$ under $K / k$, then the number of spinor genera in gen $(\Lambda, G, k)$ cannot exceed the number of spinor genera in $\operatorname{gen}\left[\psi(\Lambda), G_{K}, K\right]$.

The following lemma is immediate from the previous discussion and it will be useful later:

Lemma 2.3. If $\sigma$ is an element of the adelic group $G_{\mathbb{A}}$, the lattice $\sigma \Lambda$ belongs to the same spinor genus as $\Lambda$ if and only if $\Theta(\sigma) \in k^{*} H(\Lambda, k)$.

Let $L$ be a local field with ring of integers $\mathcal{O}_{L}$. Let $M$ be an $\mathcal{O}_{L}$-lattice in an $L$-vector space $W$. Let $G \subseteq \mathrm{GL}_{L}(W)$ be a semisimple linear algebraic group whose fundamental group is the group of $n$-roots of unity. We denote by $H(\Lambda, L)$ the preimage in $L^{*}$ of the image of the spinor norm $\theta: G^{\Lambda} \rightarrow$ $L^{*} / L^{* n}$. This is the local version of the group $H$ defined above. Note that for a global field $k$, we have $H(\Lambda, k)=\prod_{v} H_{v}(\Lambda, k)$, where $H_{v}(\Lambda, k)$ is defined as follows:

- if $v$ is non-archimedean, then $H_{v}(\Lambda, k)=H\left(\Lambda_{v}, k_{v}\right)$, where $\Lambda_{v}$ is the completion at $v$ of $\Lambda$.

- if $v$ is archimedean, then $H_{v}(\Lambda, k)$ is the preimage in $k^{*}$ of the image in $k^{*} / k^{* n}$ of the spinor norm $\theta_{v}: G_{v} \rightarrow k^{*} / k^{* n}$.

This is consistent with the convention $\Lambda_{v}=V_{v}$ for archimedean $v$. For simplicity of notation, we say that the norm principle is satisfied at the place $w$ of $K$ lying over $v$ if

$$
N_{K_{w} / k_{v}}\left[H_{w}(\psi(\Lambda), K)\right] \subseteq H_{v}(\Lambda, k) .
$$

With this notation, the norm principle is satisfied if and only if it is satisfied at every local place.

EXAMPLE 1. (The canonical extension) The simplest extension rule is $\psi(\Lambda)=\Lambda \otimes_{\mathcal{O}} \mathcal{O}_{K}$. This rule is defined for every lattice and for any semisimple group $G$. In case that $G$ is an orthogonal group the norm principle is knows to hold for all field extensions when the lattice is unimodular of rank

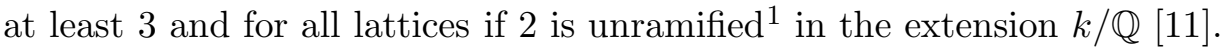

\footnotetext{
${ }^{1}$ C. N. Beli [3] has given a formula to compute the image of the spinor norm in all cases. As far as we know, this result has not yet been applied to the extension problem.
} 
ExAmple 2. (Extension rules of maximal orders) Let $V=A$ be a central simple algebra of dimension $n^{2}$ over $k$ and Let $G=A^{*} / k^{*}$ be the automorphism group of $A$. If the localization $A_{k_{v}}$ is isomorphic to $\mathbb{M}_{t}\left(A^{\prime}\right)$, where $A^{\prime}$ is a division algebra of dimension $d^{2}=d(k, v)^{2}$, then $H_{v}(\mathfrak{A}, k)=\mathcal{O}_{v}^{*} k_{v}^{* d}$ for any maximal order $\mathfrak{A}$ of $A$. It follows that if $\psi$ is an extension rule from the set of maximal orders of $A$ to the set of maximal orders of $A_{K}$, then the norm principle is satisfied provided that $d(k, v)$ divides $f\left(K_{w} / k_{v}\right) d(K, w)$ for every $w$, where $f$ denotes the residue class degree. Since $d(k, v)$ always divides $\left[K_{w}: k_{v}\right] d(K, w)$, this is the case for example when the ramification degree $e\left(K_{w} / k_{v}\right)$ is relatively prime to $d(k, v)$ for every $w$.

ExAMPLE 3. (Extension from $\mathfrak{D}$ to $\mathfrak{D}^{\prime}$ ) Let $D$ be a central simple algebra over $k$ and let $V$ be an $D$-module. Let $\mathfrak{D}$ be an order in $D$ and let $\mathfrak{D}^{\prime}$ be an order of $D_{K}$ containing $\mathfrak{D}$. Let $G$ be a group of $D$-automorphisms of $V$. Then there is an extension rule $\psi$ such that for any $\mathfrak{D}$-lattice $\Lambda$, the lattice $\psi(\Lambda)$ is the $\mathfrak{D}^{\prime}$-lattice generated by $\Lambda$, where we consider $D$ and $V$ as subsets of $D_{K}$ and $V_{K}$ respectively.

In this paper we are concerned with Example 3. As far as we know it is still unknown wether there are counterexamples to the norm principle for the canonical extension.

\section{§3. Skew-hermitian forms and matrix algebras}

In all that follows, $k$ is a local or number field, $D$ is a quaternion algebra over $k$, with standard involution $\sigma \mapsto \bar{\sigma}$, and $\mathfrak{D}$ is a maximal order in $D$. If $V$ is a $D$-module, a skew-hermitian form on $V$ is a map $h: V \times V \rightarrow V$, $D$-linear on the first variable and satisfying $h(v, w)=-\overline{h(w, v)}$. We also say that $(V, h)$ is a skew-hermitian space over $D$. An isometry between two spaces $(V, h)$ and $\left(V^{\prime}, h^{\prime}\right)$ is an invertible $D$-linear function $f: V \rightarrow V^{\prime}$ such that $h(v, w)=h^{\prime}[f(v), f(w)]$ for any pair of vectors $v$ and $w$ in $V$. Let $\mathfrak{D}$ be an order in $D$. Any skew-hermitian form $h$ on $n$ variables with coefficients in $\mathfrak{D}$, as defined in the introduction, can be viewed as a form on the $\mathfrak{D}$ module $\mathfrak{D}^{n}$ and can be extended uniquely to $V=D^{n}$. Let $h^{\prime}$ be another skew-hermitian form on $n$ variables with coefficients in $\mathfrak{D}$ such that there exists a isometry $f$ between the skew-hermitian spaces $\left(D^{n}, h\right)$ and $\left(D^{n}, h^{\prime}\right)$. Then $\Lambda=f^{-1}\left(\mathfrak{D}^{n}\right)$ is a lattice in $D^{n}$ with a $\mathfrak{D}$-basis $\left\{f^{-1}\left(e_{1}\right), \ldots, f^{-1}\left(e_{n}\right)\right\}$. As $h^{\prime}\left(e_{t}, e_{s}\right)=h\left[f^{-1}\left(e_{t}\right), f^{-1}\left(e_{s}\right)\right]$, the forms $h$ and $h^{\prime}$ are in the same class if and only if there exists a $\mathfrak{D}$-basis $\left\{v_{1}, \ldots, v_{n}\right\}$ of $\Lambda$ such that $h^{\prime}\left(e_{t}, e_{s}\right)=$ 
$h\left(v_{t}, v_{s}\right)$. We say that lattices $M$ and $L$ are equivalent if there is an isometry of $(V, h)$ sending $M$ to $L$. It follows that $h$ and $h^{\prime}$ are in the same class if and only if the lattice $\Lambda$ is equivalent to $\mathfrak{D}^{n}$. A similar argument proves that $h$ and $h^{\prime}$ are in the same genus if the lattices $\Lambda$ and $\mathfrak{D}^{n}$ are locally equivalent at all places. Conversely, any free lattice $\Lambda$ defines, once a $\mathfrak{D}$-basis is chosen, a form $h^{\prime}$ that is in the same class or genus of $h$ if and only if $\Lambda$ is in the same class or genus of $\mathfrak{D}^{n}$. It follows that clasifying forms is equivalent to clasifying free lattices. In all that follows we use the language of lattices.

The special unitary group $G$ of $h$ is the group of isometries of $V$ with reduced norm 1. This group is generated by simple rotations [5]. The simple rotation $(s ; \sigma) \in G$ of axis $s$ is defined by $(s ; \sigma)(x)=x-h(x, s) \sigma^{-1} s$, whenever $s \in V$ and $\sigma \in D^{*}$ satisfy $\sigma-\bar{\sigma}=h(s, s)$. The spinor norm on $G$ is computed by the formula $\theta[(s ; \sigma)]=N(\sigma)$ where $N$ is the reduced norm on $D$ [5]. Note that $(s ; \sigma) s=\bar{\sigma} \sigma^{-1} s$. Hence, if $\operatorname{dim}_{D} V=1$, Hilbert Theorem 90 for the extension $k(\sigma) / k$ can be reformulated as follows: Any element in the special unitary group is a simple rotation. If $D$ is a division algebra, all isometries of $h$ have reduced norm 1 ([13], p. 42). It follows that two lattices are equivalent if and only if they are in the same $G$-orbit. If $D$ is not a field, then it is isomorphic to the split quaternion algebra over $k$, i.e., the ring $\mathbb{M}_{2}(k)$ with the involution

$$
\left(\begin{array}{ll}
a & b \\
c & d
\end{array}\right)^{*}=\left(\begin{array}{cc}
d & -b \\
-c & a
\end{array}\right)
$$

Let $j$ and $\epsilon$ be the matrices defined by

$$
j=\left(\begin{array}{ll}
0 & 1 \\
1 & 0
\end{array}\right), \quad \epsilon=\left(\begin{array}{ll}
1 & 0 \\
0 & 0
\end{array}\right)
$$

Let $(V, h)$ be a skew-hermitian space over $\mathbb{M}_{2}(k)$. Since $\epsilon^{2}=\epsilon, \epsilon+j \epsilon j=1$, and $\epsilon j \epsilon=0$, then $V=\epsilon V \oplus j \epsilon V$. For $s, u \in V$ set

$$
h(s, u)=\left(\begin{array}{ll}
(s, u)_{1,1} & (s, u)_{1,2} \\
(s, u)_{2,1} & (s, u)_{2,2}
\end{array}\right) .
$$

For every $x$ and $y$ in $\epsilon V$, define $\gamma(x, y)=(x, y)_{2,1}$. Then $\gamma$ is a symmetric bilinear form on the $k$-vector space $\epsilon V$. There exists an isomorphism between $G$ and the special orthogonal group of $\gamma$ sending an element of $G$ to its restriction to $\epsilon V$ ([5], Lemma 4). Furthermore, by setting $\beta=1$ in 
formula (14) of [5], we get

$$
h\left(x_{1}+j x_{2}, y_{1}+j y_{2}\right)=\left(\begin{array}{ll}
\gamma\left(x_{1}, y_{1}\right) & \gamma\left(x_{2}, y_{1}\right) \\
\gamma\left(x_{1}, y_{2}\right) & \gamma\left(x_{2}, y_{2}\right)
\end{array}\right)\left(\begin{array}{cc}
0 & 1 \\
-1 & 0
\end{array}\right)
$$

for every $x_{1}, x_{2}, y_{1}$, and $y_{2}$ in $\epsilon V$. In particular, if $u$ and $v$ are linearly independent elements in $\epsilon V$, the discriminant of $\gamma$ in the two-dimensional space generated by $u$ and $v$ is

$$
\operatorname{disc}\left[\left.\gamma\right|_{k u+k v}\right]=N[h(u+j v, u+j v)] k^{* 2} .
$$

Note that for $x$ and $y$ in $\epsilon V$ we have the formulas

$$
\epsilon(x+j y)=x, \quad \epsilon j(x+j y)=y .
$$

Until the end of Section 3 , let $k$ be a local field, and let $\mathfrak{D}_{0}$ be the maximal order $\mathbb{M}_{2}(\mathcal{O})$. Let $\Lambda$ be a $\mathfrak{D}_{0}$-lattice. Since $j$ is a unit in $\mathfrak{D}_{0}$, we have $\Lambda=\epsilon \Lambda \oplus j \epsilon \Lambda$. Therefore, if $\left\{s_{1}, \ldots, s_{2 n}\right\}$ is a $\mathcal{O}$-basis of $\epsilon \Lambda$, then $\left\{s_{1}+\right.$ $\left.j s_{n+1}, \ldots, s_{n}+j s_{2 n}\right\}$ is a $\mathfrak{D}_{0}$-basis of $\Lambda$. By ([14], §92:1) and ([14], §93:15), the $\mathcal{O}$-lattice $\epsilon \Lambda$ has an orthogonal decomposition, $\epsilon \Lambda=\Lambda_{1} \perp \cdots \perp \Lambda_{m}$ into lattices of rank 2 . Hence, $\Lambda=\left(\Lambda_{1} \oplus j \Lambda_{1}\right) \perp \cdots \perp\left(\Lambda_{m} \oplus j \Lambda_{m}\right)$. Since $k$ is a local field, the ring $\mathcal{O}$ is a principal ideal domain. Therefore, if for $t=1, \ldots, m$ we set $\Lambda_{t}=\mathcal{O} x_{t}+\mathcal{O} y_{t}$, it holds that $\Lambda_{t} \oplus j \Lambda_{t}=\mathfrak{D}_{0}\left(x_{t}+j y_{t}\right)$. Since all maximal orders in a local central simple algebra are conjugate, next proposition follows:

Proposition 3.1. If $\mathfrak{D}$ is a maximal order in the local split quaternion algebra $\mathbb{M}_{2}(k)$, then any skew-hermitian $\mathfrak{D}$-lattice has an orthogonal basis.

By Theorem 7 in [5], the restriction to $\epsilon V$ of an element $g$ of $G$ stabilizes the lattice $\epsilon \Lambda$ if and only if $g$ stabilizes $\Lambda$. Hence, we can identify canonically the stabilizer of $\Lambda$ in $G$ with the stabilizer of $\epsilon \Lambda$ in the orthogonal group $\operatorname{Ort}(\epsilon V, \gamma)$. Since it is known that the stabilizer of a local symmetric bilinear lattice contains an element of determinant -1 ([14], p. 241), it follows that if $k$ is a local field, two lattice are equivalent if and only if they are in the same $G$-orbit, just as in the non-split case. It follows that for a quaternion division algebra $D$ over a number field $L$, and for any maximal order $\mathfrak{D}$ of $D$, two $\mathfrak{D}$-lattices in a skew-hermitian space over $D$ are locally equivalent at all places if and only if they are in the same $G$-genus. Note that $G$ is contained in the group $S L(V, D)$ of $D$ linear functions on $V$. Since this group has strong approximation, Any two lattices in the same $G$-genus are isomorphic as $\mathfrak{D}$-modules. In particular, if one is free, so is the other. 


\section{$\S 4$. Computation of $H$ for non-dyadic split places}

In this section we give some spinor norm computations that we use to prove the main results. In all of this section $k$ is a non-dyadic local field with ring of integers $\mathcal{O}$, uniformizing parameter $\pi$, and a valuation $\nu$ such that $\nu(\pi)=1$. Assume $D=\mathbb{M}_{2}(k)$ and $\mathfrak{D}=\mathbb{M}_{2}(\mathcal{O})$. We identify the unitary group $G$ of the skew-hermitian form $h$ with the orthogonal group $\operatorname{Ort}(\gamma)$ and the stabilizer $G^{\Lambda}$ of the lattice $\Lambda$ with $\operatorname{Ort}(\gamma)^{\epsilon \Lambda}$ in the notations of last section. A pure quaternion $a$ in $\mathfrak{D}$ is good if the ring of integers $\mathcal{O}_{k(a)}$ of the algebra $k(a)$ is contained in $\mathfrak{D}$. Let $a=h(s, s)$, for some element $s \in \Lambda$. Assume that $\nu[N(a)]=2 t$ is even. Then $\pi^{-t} a \in \mathcal{O}_{k(a)}^{*}$. Assume that $a$ is good, so that $\pi^{-t} a \in \mathfrak{D}^{*}$. Then $\epsilon(\mathfrak{D} s)$ is a modular symmetric bilinear lattice of scale $\left(\pi^{t}\right)$. Next result follows from Theorem 3 in [12]:

Proposition 4.1. Assume that $\Lambda=\mathfrak{D} s_{1} \perp \cdots \perp \mathfrak{D} s_{n}$, where $a_{m}=$ $h\left(s_{m}, s_{m}\right) \in \mathfrak{D}$ for $m=1, \ldots, n$. Assume also that $\nu\left[N\left(a_{1}\right)\right], \ldots, \nu\left[N\left(a_{n}\right)\right]$ are all even and congruent modulo 4 , and that $a_{1}, \ldots, a_{n}$ are all good. Then, $H(\Lambda, k)=\mathcal{O}^{*}\left(k^{*}\right)^{2}$.

Proposition 4.2. Let $\alpha$ be a non-unit in $\mathcal{O}_{k}$. Let $q$ be a pure quaternion satisfying $q^{2}=\alpha$. Assume that $\Lambda=\mathfrak{D} s_{1} \perp \cdots \perp \mathfrak{D} s_{n}$, where $h\left(s_{m}, s_{m}\right)=(-\alpha)^{t_{m}} q$ for $m=1, \ldots, n$. Assume also that either of the following conditions hold:

- $t_{m+1} \geq t_{m}+2$ for $m=1, \ldots, n-1$, and $\nu(\alpha)$ is odd.

- $t_{m+1} \geq t_{m}+1$ for $m=1, \ldots, n-1, \nu(\alpha)$ is even, and $\alpha \notin k^{* 2}$.

Then $H(\Lambda, k)=N\left(k(q)^{*}\right)$.

Proof. We can assume that the symmetric bilinear lattice $\epsilon\left(\mathfrak{D} s_{1}\right)$ is diagonal, whence by (3) we have $q=\left(\begin{array}{ll}0 & y \\ z & 0\end{array}\right)$, for some $y$ and $z$ in $\mathcal{O}$ satisfying $y z=\alpha$. Conjugating by $j=\left(\begin{array}{ll}0 & 1 \\ 1 & 0\end{array}\right)$ if needed, we may assume $\nu(y) \geq \nu(z)$, so that $2 \nu(z) \leq \nu(\alpha)$. It follows that

$$
\epsilon \mathfrak{D} s_{m} \cong\left\langle(-\alpha)^{t_{m}} z\right\rangle \perp\left\langle(-\alpha)^{t_{m}+1} z^{-1}\right\rangle .
$$

If $\nu(\alpha)$ is even, it follows that all Jordan components of $\epsilon \Lambda$ are modular lattices with scales of the same parity, and the result follows from Theorem 3 in [12] and $N\left(k(q)^{*}\right)=\mathcal{O}^{*}\left(k^{*}\right)^{2}$. Assume now that $\nu(\alpha)$ is odd, so that $2 \nu(z)<\nu(\alpha)$. If a lattice $\left\langle(-\alpha)^{t} z\right\rangle$ has the same scale as 
a lattice $\left\langle(-\alpha)^{t^{\prime}+1} z^{-1}\right\rangle$ we have $t \nu(\alpha)+\nu(z)=\left(t^{\prime}+1\right) \nu(\alpha)-\nu(z)$ and therefore $\left(t-t^{\prime}\right) \nu(\alpha)=\nu(\alpha)-2 \nu(z)$. It follows that $t-t^{\prime}=1$. By the condition on the exponents $t_{m}$, the symmetric bilinear lattice $\epsilon \Lambda$ has all its Jordan components are of the form $\left\langle(-\alpha)^{t} z\right\rangle$ or $\left\langle(-\alpha)^{t} z^{-1}\right\rangle$. Hence $H(\Lambda, k)=\left(k^{*}\right)^{2} \cup(-\alpha)\left(k^{*}\right)^{2}=N\left(k(q)^{*}\right)$ by Theorem 3 in [12].

Proposition 4.3. Let $i$ a quaternion such that $i^{2}=\pi$ is a uniformizing parameter of $k$. Let $\Lambda$ be the skew-hermitian lattice $\Lambda=\langle i\rangle \perp\langle\pi i\rangle$. Then $H(\Lambda, k)=k^{*}$.

Proof. Consider the skew-hermitian lattice $L=\langle i\rangle$. The symmetric bilinear lattice $\epsilon L$ has discriminant $-\pi$ and therefore, it must have a Jordan decomposition of the form $\epsilon L \cong\langle u\rangle \perp\langle-u \pi\rangle$, where $u$ is a unit. It follows that

$$
\epsilon \Lambda \cong\langle u\rangle \perp\langle-u \pi\rangle \perp\langle u \pi\rangle \perp\left\langle-u \pi^{2}\right\rangle .
$$

The result follows from the relation $\langle\pi\rangle \perp\langle-\pi\rangle \cong\langle\Delta \pi\rangle \perp\langle-\Delta \pi\rangle$ and Theorem 3 in [12].

\section{$\S 5$. Norm principle for non-dyadic places}

In all of Section $5 k, \pi, \mathcal{O}$, and $\nu$ are as in last section. Let $D$ be a quaternion division algebra over $k$ with a maximal order $\mathfrak{D}$. Let $\Lambda$ be a $\mathfrak{D}$-lattice in a skew-hermitian space $(V, h)$ with unitary group $G$. Let $\left\{s_{1}, \ldots, s_{n}\right\}$ be an orthogonal $\mathfrak{D}$-basis of $\Lambda$. Let $a_{m}=h\left(s_{m}, s_{m}\right)$, for $1 \leq$ $m \leq n$. It is proved in [5] that if the image of the spinor norm is not the whole of $k^{*}$, then it has the form $N_{L / k}\left(L^{*}\right)$, for the quadratic extension $L=k\left(a_{1}\right)$. This is the case in any of the following cases:

1. $\nu\left[N\left(a_{m}\right)\right]$ is even for all $m \in\{1, \ldots, n\}$.

2. $\nu\left[N\left(a_{m}\right)\right]<\nu\left[N\left(a_{m+1}\right)\right]$ and $N\left(a_{m}\right) \equiv N\left(a_{m+1}\right)\left(\bmod k^{* 2}\right)$ for all $m \in\{1, \ldots, n-1\}$.

If we have two finite extensions $L$ and $K$ of $k$, the above computation and the contention $N_{L / k}\left(L^{*}\right) \supseteq N_{K / k} \circ N_{K L / K}\left[(K L)^{*}\right]$ imply next result:

Proposition 5.1. Let $K / k$ be an odd degree extension of non-dyadic local fields. Let $\mathfrak{D}^{\prime}$ be a maximal order of $D_{K}$ containing $\mathfrak{D}$. Let $\psi$ be the extension rule defined in Example 3. Then $\psi$ satisfy the norm principle for $\Lambda$ under $K / k$. 
If $K / k$ is an even extension, and hence $D_{K}$ is a matrix algebra, we need the results in last section to study the norm principle.

LEMmA 5.2. Let $\beta$ be an element of $k$ whose valuation $\nu(\beta)$ is odd. If $N\left(q_{1}\right)=N\left(q_{2}\right)=\beta$ for $q_{1}, q_{2} \in D$, then $q_{1}=u q_{2} \bar{u}$ for some $u \in \mathfrak{D}^{*}$.

Proof. Multiplying by elements in the center, we can assume that $\nu(\beta)=1$. The space of pure quaternions is a quadratic space of dimension 3 with the norm and the set $\mathfrak{D}^{0}$ of pure quaternions in $\mathfrak{D}$ is a lattice in this space. By Witt's Theorem for non-dyadic local lattices [14] there is an element of the special orthogonal group of the lattice that sends $q_{1}$ to $q_{2}$. Since any element in the special orthogonal group of the space of pure quaternions is a conjugation, the elements $q_{1}$ and $q_{2}$ are conjugate. Write $q_{1}=r q_{2} r^{-1}$. Observe that $k\left(q_{2}\right) / k$ is ramified by the condition on $\beta$. Multiplying $r$ by an element in $k\left(q_{2}\right)$, we can assume $r$ is a unit. Hence, replacing $q_{2}$ by $r q_{2} \bar{r}$, we can assume $q_{1}=\eta q_{2}$, where $\eta \in \mathcal{O}^{*}$. If $\eta$ is a square, there is nothing left to prove. We can assume that $\eta$ is a non square unit. Since the Hilbert symbol $(\eta,-\beta)$ defines the algebra $D$, there exists a pure quaternion $q_{3} \in D$ such that $q_{3}^{2}=\eta$, and $q_{3} q_{2}=-q_{2} q_{3}$. It follows that $q_{3} q_{2} \overline{q_{3}}=\eta q_{2}$. The result follows.

Proposition 5.3. Let $K / k$ be an even-degree extension. Let $\mathfrak{D}^{\prime}$ be a maximal order of $D_{K}$ containing $\mathfrak{D}$. If the extension rule $\psi$ of Example 3 does not satisfy the norm principle for the lattice $\Lambda$ under the extension $K / k$, then the following conditions hold:

- The valuations $\nu\left[N\left(a_{1}\right)\right], \ldots, \nu\left[N\left(a_{n}\right)\right]$ are all odd.

- $\nu\left[N\left(a_{m}\right)\right]<\nu\left[N\left(a_{m+1}\right)\right]$ for all $m=1, \ldots, n-1$.

- $N\left(a_{m}\right) \equiv N\left(a_{r}\right)\left(\bmod k^{* 2}\right)$ for all $r, m=1, \ldots, n$.

- The ramification degree $e(K / k)$ is odd.

- $\nu\left[N\left(a_{t+1}\right)\right]=\nu\left[N\left(a_{t}\right)\right]+2$, for some $t$.

Proof. If $H(\Lambda, k)=k^{*}$ there is nothing to prove. According to [5] this holds except in cases 1 and 2 at the beginning of this section.

1. In this case $H(\Lambda, k)=\mathcal{O}^{*}\left(k^{*}\right)^{2}$. Let $\Delta$ be a non-square unit in $k$. If $\Delta$ is not a square in $K$, we claim that Proposition 4.1 applies. First, we need to prove that $a_{1}, \ldots, a_{n}$ are good. Note that $\mathcal{O}_{k\left(a_{m}\right)}=\mathcal{O}(\kappa)$, where $\kappa \in \mathfrak{D}$ satisfies $\kappa^{2}=\Delta$, hence $\mathcal{O}_{K\left(a_{m}\right)}=\mathcal{O}_{K}(\kappa) \subseteq \mathfrak{D}^{\prime}$. The 
congruence hypotheses follows since the ramification degree $e(K / k)$ is even. Now the proof goes as for Proposition 5.1. Therefore, we can assume that $\Delta$ is a square in $K$. In this case $K$ contains an unramified quadratic extension $L$ of $k$, hence:

$$
\begin{aligned}
N_{K / k}[H(\psi(\Lambda), K)] & \subseteq N_{K / k}\left(K^{*}\right) \subseteq N_{L / k}\left(L^{*}\right) \\
& =\mathcal{O}^{*}\left(k^{*}\right)^{2}=H(\Lambda, k) .
\end{aligned}
$$

2. In this case we have $H(\Lambda, k)=N\left[k\left(a_{1}\right)^{*}\right]$. If $K\left(a_{m}\right)$ is not a field, then $k\left(a_{m}\right)$ injects in $K$ and the result follows as in (6). If $K\left(a_{m}\right)$ is a field there are three possibilities depending on the ramification degree $e(K / k)$.

- If $e(K / k)$ is odd and for some $m$ we have $\nu\left[N\left(a_{m+1}\right)\right]=$ $\nu\left[N\left(a_{m}\right)\right]+2$, then we are in the exceptional case.

- Assume that $e(K / k)$ is odd, and $\nu\left[N\left(a_{m+1}\right)\right]>\nu\left[N\left(a_{m}\right)\right]+2$ for all $m=1, \ldots, n-1$. Replacing $a_{m}$ by $u_{m} a_{m}$, where $u_{m} \in$ $\mathcal{O}^{*} \subseteq \mathcal{O}_{K}^{* 2}$, if needed, we may assume $N\left(a_{m}\right)=(-\alpha)^{2 t_{m}+1}$, for a suitable uniformizing parameter $\alpha$ of $k$. By Lemma 5.2 we can assume $a_{m}=(-\alpha)^{t_{m}} q$, where $q$ is as in Proposition 4.2, and therefore $H[\psi(\Lambda), K] \subseteq N\left[K(q)^{*}\right]$. Now the result follows as for Proposition 5.1.

- If $e(K / k)$ is even, then $N_{K / k}\left(\mathcal{O}_{K}^{*} K^{* 2}\right) \subseteq k^{* 2}$. On the other hand, by the same argument as above we have $H[\psi(\Lambda), K] \subseteq$ $N\left[K(q)^{*}\right]=\mathcal{O}_{K}^{*} K^{* 2}$.

ExAmPle. Assume $K / k$ is an unramified quadratic extension. By Proposition 4.3, if $N(i)=-\pi$, the lattice $\Lambda=\langle i\rangle \perp\langle\pi i\rangle$ has full spinor norm over $K$, but $N_{K / k}\left(K^{*}\right)=\mathcal{O}^{*}\left(k^{*}\right)^{2}$ is not contained in $N\left[k(i)^{*}\right]=H(\Lambda, k)$.

Since the choice of the order $\mathfrak{D}^{\prime}$ in $D_{K}$ is not unique, if $e(K / k)$ is odd and bigger than 1 , some choices of $\mathfrak{D}^{\prime}$ might still make the norm principle hold. One way to accomplish this is by lifting $\mathfrak{D}$ first to some ramified odd degree subextension of $K / k$.

\section{§6. Proof of Theorems 3, 4, and 5}

For the following proposition, notations are as in Section 5, except that here $k$ is a dyadic field and $\Delta \in k$ is a unit of maximal quadratic defect. If $\Lambda$ is diagonalizable, we assume that $\Lambda=\mathfrak{D} s_{1} \perp \cdots \perp \mathfrak{D} s_{n}$ and $a_{m}=$ $h\left(s_{m}, s_{m}\right)$. Next result follows as Proposition 5.1 using the tables in [2]. 
Proposition 6.1. Let $K / k$ be an odd degree extension of dyadic local fields. Let $\mathfrak{D}^{\prime}$ be the maximal order of $D_{K}$. Let $\psi$ be the extension rule defined in Example 3. Then $\psi$ satisfy the norm principle for $\Lambda$ under $K / k$ except maybe in the following cases:

- 2 is not a prime in $k$ and $\Lambda$ is not diagonalizable.

- $\Lambda$ is diagonalizable. The norms of the elements $a_{m}$ have the form $N\left(a_{m}\right)=\lambda_{m}^{2} \beta$, for a fixed element $\beta \in k$ that is not in the same square class as $\Delta$, and elements $\lambda_{1}, \ldots, \lambda_{n} \in k$ such that $\nu\left(\lambda_{m}\right)+\varepsilon \leq \nu\left(\lambda_{m+1}\right)$ for $m=1, \ldots, n-1$, where

$$
\varepsilon= \begin{cases}1 & \text { if } \nu(\beta) \text { is even } \\ \nu(4) & \text { if } \nu(\beta) \text { is odd }\end{cases}
$$

Furthermore, for some $m$ we have $\nu\left(\lambda_{m}\right)+\nu(16) \geq \nu\left(\lambda_{m+1}\right)$.

As noted in Section 3, there is a one to one correspondence between classes or genera of skew-hermitian forms defined over $\mathfrak{D}$ that are equivalent to $h$ over $D$ and $G$-classes or $G$-genera of free $\mathfrak{D}$-lattices in $(V, h)$. Therefore, to prove Theorems 3,4 , and 5 , we can use the language of lattices.

Proof of Theorem 5. Assume $K / k$ is a Galois extension of odd degree. Then $\operatorname{spn}[\psi(\Lambda)]=\operatorname{spn}[\psi(L)]$ implies $\operatorname{spn}(\Lambda)=\operatorname{spn}(L)$, provided that the norm principle is satisfied by Proposition 2.1. As noted in Section 2, the norm principle holds if it holds locally at all places. The Galois condition implies that all local degrees are odd. At non-dyadic places $v$ where the algebra $D_{v}$ does not split, the norm principle holds by Proposition 5.1. At non-dyadic places $v$ where the algebra $D_{v}$ splits, we can use the correspondence between stabilizers of skew-hermitian lattices $\Lambda$ and stabilizers of orthogonal lattices $\epsilon \Lambda$ described in Section 3 to reduce the problem to proving the norm principle for orthogonal lattices. The norm principle follows immediately from Theorem 3 in [12], since quadratic classes do not change under an odd degree extension. Therefore, we are reduced to prove the norm principle at a dyadic place $v$. If $v$ is in case (1), as before, we are reduced to proving the norm principle for orthogonal lattices, which is done in the Main Lemma for the proof of Theorem 4.1 in [8]. It follows from last proposition that the norm principle is satisfied in case (3). Case (2) is trivial. 
Proof of Theorem 4. We need to prove that the localization $\Lambda_{v}$ at every dyadic place $v$ satisfies one of the conditions 1,2 , or 3 in Theorem 5 . If the local algebra $D_{v}$ splits, condition 1 is satisfied, so we can assume that $D_{v}$ does not split. By Lemma 5.2 in [2], every unimodular $\mathfrak{D}_{v}$-lattice has an orthogonal basis, whence $\Lambda_{v}$ is not in the first exceptional case in condition 3. It is clear that it is not in any of the others.

Proof of Theorem 3. It suffices to prove that the norm principle holds locally at all finite places. Because of Theorem 4.1 in [8] and the fact that spinor norm for a lattice $\Lambda$ is the same as for the corresponding symmetric bilinear lattice $\epsilon \Lambda$ as in Section 3, it suffices to consider places $v$ that are ramified for $D$. Let $q \in \mathfrak{D}_{v}$ be a pure quaternion such that $q^{2}=\Delta$ is a unit of maximal quadratic defect. If $v$ is non-dyadic, this is equivalent to say that $\Delta$ is a non-square unit. In any case, this is equivalent to say that the residue class degree $f\left[k_{v}(q) / k_{v}\right]$ equals 2 , or equivalently $q$ is a unit in $\mathfrak{D}^{*}$ satisfying $q=\omega-\bar{\omega}$ for some $\omega \in \mathfrak{D}$. Assume first $\Lambda=\langle q\rangle \perp \cdots \perp\langle q\rangle$. Now, it follows from the tables in [2] and Theorem 4 in [5] that $H(\Lambda, k)=\mathcal{O}^{*} k^{* 2}$. Now let $K_{V} / k_{v}$ be any local extension. If $\left[K_{V}: k_{v}\right]$ is odd, the extension of $\Lambda$ to $\mathcal{O}_{K_{V}}$ is also of the form $\langle q\rangle \perp \cdots \perp\langle q\rangle$, where $q=\omega-\bar{\omega}$. It follows that $H\left[\psi(\Lambda), K_{V}\right]=\mathcal{O}_{K_{V}} K_{V}^{* 2}$. Assume now that $\left[K_{V}: k_{v}\right]$ is even, so that $D_{K_{V}}$ is isomorphic to a matrix algebra. If $L=\langle q\rangle$ is a lattice over $\mathfrak{D}_{K_{V}}$, it follows from (4) that $\epsilon L$ is a binary unimodular lattice, whence it has a Gram Matrix of the form ( $\left.\begin{array}{ll}z & 1 \\ 1 & y\end{array}\right)$. By (3) we can choose the isomorphism so that $q$ corresponds to a matrix of the form $\left(\begin{array}{ll}-1 & z \\ -y & 1\end{array}\right)$, where the fact that $q=\omega-\bar{\omega}$ for an integer $\omega$ proves that $y$ and $z$ are in the ideal $2 \mathcal{O}_{K_{V}}$. Therefore, in the notations of Section 3, the lattice $\epsilon \psi(\Lambda)$ has the form $\left\langle\begin{array}{ll}z & 1 \\ 1 & y\end{array}\right\rangle \perp \cdots \perp\left\langle\begin{array}{ll}z & 1 \\ 1 & y\end{array}\right\rangle$. By Lemma 1 in [10], we have $H[\psi(\Lambda), K]=\mathcal{O}_{K}\left(K^{*}\right)^{2}$, whence the norm principle holds. If $\Lambda \neq\langle q\rangle \perp \cdots \perp\langle q\rangle$, then $v$ must be dyadic and the tables in [2] prove that $H(\Lambda, k)=k^{*}$ whence norm principle is immediate.

\section{§7. Proof of Theorems 1 and 2}

Let $D$ be a quaternion algebra over $\mathbb{Q}$ generated by orthogonal skewhermitian elements $j$ and $i$, satisfying $j^{2}=-1$ and $i^{2}=p q$, where $p$ and $q$ are positive primes satisfying $p, q \equiv 3(\bmod 4)$ and $p q \equiv 5(\bmod 8)$. This quaternion algebra splits everywhere except at the places $p$ and $q$. Let $\mathfrak{D}$ be a maximal order containing $\omega=(1+i) / 2$ and $j$. Consider the skewhermitian form

$$
h\left(x_{1}, x_{2} ; y_{1}, y_{2}\right)=x_{1} i \overline{y_{1}}+p q x_{2} i \overline{y_{2}},
$$


and let $\Lambda$ be $\mathfrak{D} \times \mathfrak{D}$ with the form $h$. As noted in Section 3 , there is a correspondence between classes of free $\mathfrak{D}$-lattices in the genus of $\Lambda$ and classes of forms in the genus of $h$. Since the group $\operatorname{SL}_{2}(D)$ has strong approximation and contains the unitary group $G$ of $h$, every lattice in the genus of $\Lambda$ is free. Since $D_{\mathbb{R}}$ is a matrix algebra, and $N(i)<0$, so that the symmetric bilinear lattice $\epsilon\langle i\rangle$ is indefinite, the local image of the spinor norm at $\infty$ is $H(V, \mathbb{R})=\mathbb{R}^{*}$. For $v=2$, we use $i=\omega-\bar{\omega}$ to prove that $\epsilon\langle i\rangle$ is even as in last section. From the computations in [5], and Section 4, we complete the following table:

$$
H_{v}(\Lambda, \mathbb{Q})= \begin{cases}N\left[\mathbb{Q}_{v}(\sqrt{p q})^{*}\right] & \text { if } v=p, q \\ \mathbb{R}^{*} & \text { if } v=\infty \\ \mathbb{Z}_{v}^{*} \mathbb{Q}_{v}^{* 2} & \text { if } v \neq p, q, \infty .\end{cases}
$$

It follows that $\Sigma_{\mathbb{Q}}=\mathbb{Q}(\sqrt{p q})$. In particular, the genus of $\Lambda$ has two spinor genera. The representatives of both spinor genera are $\Lambda$ and $\sigma \Lambda$, where $\sigma_{r}=I d$ for $r \neq p$ and $\sigma_{p}$ is any rotation with spinor norm -1 .

Proof of Theorem 2. If $K=\mathbb{Q}(\sqrt{-1})$, then $D_{K}$ is a matrix algebra. Therefore, Proposition 4.3 shows that $H(\Lambda, K, v)=K_{v}^{*}$ at both $v=p$ and $v=q$. Since it is unimodular everywhere else, it follows that $\Sigma_{K} / K$ is unramified everywhere and therefore $\Sigma_{K}=K$. Therefore, the spinor class number decreases in spite of the fact that $K \cap \Sigma_{k}=k$.

Proof of Theorem 1 . Find a cubic extension $K / \mathbb{Q}$, so that $(p)=\wp_{1} \wp_{2}$ and $(q)=\mathfrak{q}_{1} \mathfrak{q}_{2}$ where $\wp_{1}, \wp_{2}, \mathfrak{q}_{1}$, and $\mathfrak{q}_{2}$ are prime ideals in $K$ satisfying the following conditions:

$$
\left[K_{\wp_{1}}: \mathbb{Q}_{p}\right]=\left[K_{\mathfrak{q}_{1}}: \mathbb{Q}_{q}\right]=1, \quad\left[K_{\wp_{2}}: \mathbb{Q}_{p}\right]=\left[K_{\mathfrak{q}_{2}}: \mathbb{Q}_{q}\right]=2 .
$$

It suffices to choose $p, q$, and $K$ in such a way that the idele having coordinates -1 at $\wp_{1}$ and $\wp_{2}$ and 1 everywhere else is in $H(\Lambda, K) K^{*}$. Since $H\left(\Lambda, K, \wp_{2}\right)=K_{\wp_{2}}^{*}$ and $H\left(\Lambda, K, \mathfrak{q}_{2}\right)=K_{\mathfrak{q}_{2}}^{*}$ by Proposition 4.3 , this is immediate if $K$ has a unit that is a local square at $\mathfrak{q}_{1}$ but not at $\wp_{1}$. Let $\alpha$ be a root of $x^{3}+x+1=0$. Then $\alpha$ is a unit of the cubic field $K=\mathbb{Q}(\alpha)$. A prime $p$ has the above decomposition in $K$ if and only if $x^{3}+x+1=0$ has exactly one simple root in $\mathbb{Z} / p \mathbb{Z}$. Note that 2 is the only root of $x^{3}+x+1=0$ in $\mathbb{Z} / 11 \mathbb{Z}$ and 4 is the only root of $x^{3}+x+1=0$ in $\mathbb{Z} / 23 \mathbb{Z}$. Set $p=11$ and $q=23$. We conclude that $\alpha \equiv 2\left(\bmod \wp_{1}\right)$ and $\alpha \equiv 4\left(\bmod \mathfrak{q}_{1}\right)$. Since 4 is a square in $\mathbb{Z} / 23 \mathbb{Z}$ and 2 is not a square in $\mathbb{Z} / 11 \mathbb{Z}$, the result follows. 


\title{
REFERENCES
}

[1] L. E. Arenas-Carmona, Applications of spinor class fields: embeddings of orders and quaternionic lattices, Ann. Inst. Fourier, 53 (2003), 2021-2038.

[2] L. E. Arenas-Carmona, Spinor norm for local skew-hermitian forms, Contemporary Math., 344 (2004), 19-29.

[3] C. N. Beli, Integral spinor norm groups over dyadic local fields, J. Number Th., 102 (2003), 125-182.

[4] J. W. Benhamand and J. S. Hsia, On exceptional spinor representations, Nagoya Math. J., 87 (1982), 247-260.

[5] S. Böge, Spinorgeschlechter schiefhermitescher Formen, Arch. Math., XXI (1970), $172-184$.

[6] K. S. Brown, Cohomology of groups, Springer-Verlag, New York, 1994.

[7] A. G. Earnest and J. S. Hsia, Spinor norms of local integral rotations II, Pacific J. Math., 61 (1975), 71-86; also ibid. 115 (1984), 493-494.

[8] A. G. Earnest and J. S. Hsia, Spinor genera under field extensions II: 2 unramified in the bottom field, Am. Journal of Math., 100 (1978), 523-538.

[9] D. R. Estes and J. S. Hsia, Spinor genera under field extensions IV: Spinor class fields, Japanese J. Math., 16 (1990), 341-350.

[10] J. S. Hsia, Spinor norms of local integral rotations I, Pacific J. of Math., 57 (1975), 199-206.

[11] J. S. Hsia, Arithmetic of indefinite quadratic forms, Contemporary Math., 249 (1999), 1-15.

[12] M. Kneser, Klassenzahlen indefiniter quadratischen Formen in drei oder mehr Veränderlichen, Arch. Math., VII (1956), 323-332.

[13] M. Kneser, Lectures on Galois cohomology of classical groups, TATA Institute of Fundamental Research, Bombay, 1969.

[14] O. T. O'meara, Introduction to quadratic forms, Academic Press, New York, 1963.

[15] V. P. Platonov, A. A. Bondarenko and A. S. Rapinchuk, Class numbers and groups of algebraic groups, Math. USSR Izv., 14 (1980), 547-569.

[16] V. P. Platonov and A. S. Rapinchuk, Algebraic groups and number theory, Academic Press, Boston, 1994.

[17] W. Scharlau, Quadratic and Hermitian forms, Springer Verlag, Berlin, 1985.

\author{
Universidad de Chile \\ Facultad de Ciencias \\ Casilla 653, Santiago \\ Chile
}

learenas@uchile.cl 